\title{
Anatomic double-bundle medial patellofemoral ligament reconstruction with aperture fixation using an adjustable- length loop device: a 2-year follow-up study
}

Jae-Ang Sim¹, Jin-Kyu Lim² and Byung Hoon Lee ${ }^{2^{*}}$

\begin{abstract}
Background: To assess the clinical availability of an adjustable-length loop device for use in the double-bundle technique with aperture fixation at the patella and femur during anatomic double-bundle medial patellofemoral ligament reconstruction (DB-MPFLR) for recurrent patellar dislocation.
\end{abstract}

Methods: We retrospectively investigated 11 patients (12 knees) with recurrent patellar dislocation who underwent anatomic DB-MPFLR with an ipsilateral semitendinosus tendon autograft. The graft was folded in half, and its central portion was hanged using the adjustable-length loop device. Both free ends of the graft were fixed at the proximal and distal ends of the medial edge of the patella by using suture anchors, and the hanged graft loop was pulled into the femoral tunnel while maintaining equal tension on both bundles. Manual traction of the suture loops was applied to fix the graft appropriately in full range of motion (ROM) of the knee joint under arthroscopic guidance. Clinical outcomes such as re-dislocation, ROM, clinical scores (Kujala score, Lysholm score, and visual analogue scale score for anterior knee pain), and complications were assessed preoperatively and at 2 years postoperatively. Radiographic parameters indicating patellar position, including congruence angle and lateral patellofemoral angle, were measured at 4 different angles of knee flexion $\left(30^{\circ}, 45^{\circ}, 60^{\circ}\right.$, and $\left.90^{\circ}\right)$.

Results: At 4 different flexion angles of the knee joint, the preoperative congruence angle decreased significantly and the lateral patellofemoral angle increased significantly at the final follow-up $(P<0.001)$. Notably, the improvements in these angles were maintained with no significant differences at the 4 different flexion angles. None of the patients experienced subluxation or re-dislocation after surgery. The patellar instability symptoms improved, as confirmed on the basis of radiographic and other clinical outcomes.

Conclusion: New DB technique with aperture fixation at the patella and femur by using an adjustable-length loop device offers high stability with full ROM of the knee joint, can be considered as a feasible procedure and technique for recurrent patellar dislocation.

Keywords: Medial patellofemoral ligament, Patella, Recurrent patellar dislocation, Double bundle, Aperture fixation, Adjustable-length loop device

\footnotetext{
* Correspondence: oselite@naver.com

${ }^{2}$ Department of Orthopedic Surgery, Kang-Dong Sacred Heart Hospital,

Hallym University Medical School, 134-701, Gil-dong, Seoul, South Korea

Full list of author information is available at the end of the article
}

(c) The Author(s). 2018 Open Access This article is distributed under the terms of the Creative Commons Attribution 4.0 International License (http://creativecommons.org/licenses/by/4.0/), which permits unrestricted use, distribution, and reproduction in any medium, provided you give appropriate credit to the original author(s) and the source, provide a link to the Creative Commons license, and indicate if changes were made. The Creative Commons Public Domain Dedication waiver (http://creativecommons.org/publicdomain/zero/1.0/) applies to the data made available in this article, unless otherwise stated. 


\section{Background}

Recurrent patellar dislocation (RPD) is related to various pathological abnormalities [1-10]. The medial patellofemoral ligament (MPFL) provides a primary restraint against the lateral dislocation of the patella $[11,12]$, and MPFL insufficiency is considered to be the main cause of traumatic RPD or patellar instability [13]. During MPFL reconstruction, graft fixation is critical to ensure the restoration of MPFL function. Several techniques have been introduced to fix the graft to the patellar MPFL attachment site, including the patellar bone tunnel technique [14-16] and the suture anchor technique $[14,17]$.

Non-anatomic reconstruction of the MPFL can lead to non-physiologic patellofemoral pressure and abnormal patellar tracking [18]. Therefore, recent techniques for reconstruction of the medial patellofemoral complex seek to restore the identical footprint of both the patellar and femoral attachments for biomechanical matching. The anatomic attachment site and anatomic shape of the native MPFL was previously defined. Double-bundle (DB) reconstruction at the patellar side may be a reasonable method for restoring the native ligamentous morphologic and biomechanical properties [17]. Therefore, increased interest has been directed toward anatomic DB reconstruction, which replicates 2 functional bundles, to more closely restore the normal patellofemoral stability and kinematics.

Nevertheless, the biomechanical rationale of anatomic DB reconstruction is not well established [19]. During DB anterior cruciate ligament reconstruction, both the anteromedial and posterolateral bundles are stretched and loaded in the extended knee position [20]. Therefore, graft fixation in this stretched and loaded position avoids elongation of the graft, potentially facilitating early rehabilitation with full range of motion (ROM). However, the DB MPFL reconstruction (DB-MPFLR) does not take into consideration the length change pattern of the respective bundles.

The aperture fixation technique introduced by Schöttle et al. [21] may not apply the length change patterns at each knee flexion of the MPFL, a complex of functionally varying fibers, with some taut and others slack, throughout the range of knee motion [19]. Therefore, direct anatomic/aperture fixation [22] to restore the triangular form of the MPFL for anatomic reconstruction can result in uneven and non-isometric graft tensioning and might induce non-physiologic patellofemoral loads and kinematics [23] with full ROM. Furthermore, micro-motion of the graft during knee flexion-extension can increase the risk of delayed or insufficient tendon-to-bone healing [24].

We hypothesized that the adjustable-length loop device used in femoral cortical suspension systems, which are the most convenient devices for use in ligament reconstruction with soft tissue graft [25], will be applicable in anatomic DB-MPFLR, with 2 clinical benefits. First, certain reciprocal movement of the looped graft into the femoral tunnel may allow the even tension to restrain the lateral force throughout the ROM. Second, graft fixation with appropriate tension in full ROM of the knee joint can be easily achieved by manual traction using lead sutures under arthroscopic guidance. Here, we describe a DB technique with aperture fixation at the patella and femur by using an adjustable-length loop device, which offers high stability in full ROM of the knee joint.

\section{Materials and methods}

Between 2014 and 2015, 18 patients underwent surgery for the treatment of RPD depending on individual pathologic abnormalities. All surgeries were done by the same senior orthopedic surgeon. All patients who underwent surgery during this period were screened. The indication for operation was RPD (defined as at least 2 episodes of patellar dislocation despite non-operative treatment). Lateral patellar dislocations were diagnosed on the basis of history taking, physical examination, simple radiographs, computed tomography, and magnetic resonance imaging. Seven patients who required additional procedures for RPD and had various pathologic abnormalities $[1,2]$, such as bony pathologies on the femoral or tibial side, were excluded, as follows: trochlear dysplasia (Dejour classification C) $[3,4,26]$ $(n=1)$, increased tibial tuberosity to trochlear groove (TT-TG) distance $(>20 \mathrm{~mm})[4,5](n=4)$, patella alta (Insall-Salvati [IS] ratio $>1.5)$ [27] $(n=1)$, and combined genu valgum deformity (criteria: $> \pm 3^{\circ}$ mechanical femorotibial angle [MFTA] on anteroposterior long-leg weightbearing lower-extremity scanographs) [28] $(n=1)$. Patellar height, TT-TG distance, and MFTA were preoperatively assessed by the same surgeon. Patients with a minimum postoperative follow-up of 2 years were considered eligible. Eventually, a total of 11 patients (12 knees) with RPD were treated using our approach of anatomic DB-MPFLR. The current study obtained institutional review board approval (GAIRB 2017-236) before the study onset, and informed consent was obtained from all patients. The patients' demographic data are presented in Table 1.

\section{Surgical technique}

Before RPD correction, diagnostic arthroscopic examination was performed in all patients. After the completion of arthroscopy, a 2-cm-long oblique incision was performed at the pes anserinus. After incising the sartorius aponeurosis, the semitendinous tendon was harvested and used as an autograft. The usable part of the tendon needed to be at least $20 \mathrm{~cm}$ long. After the tendon was harvested using a stripper and the muscle tissue was removed, the doubled tendon diameter was determined and both ends were whip stitched using an absorbable braided suture over a length of $15 \mathrm{~mm}$. The graft was then folded in half, and its central portion was hanged 


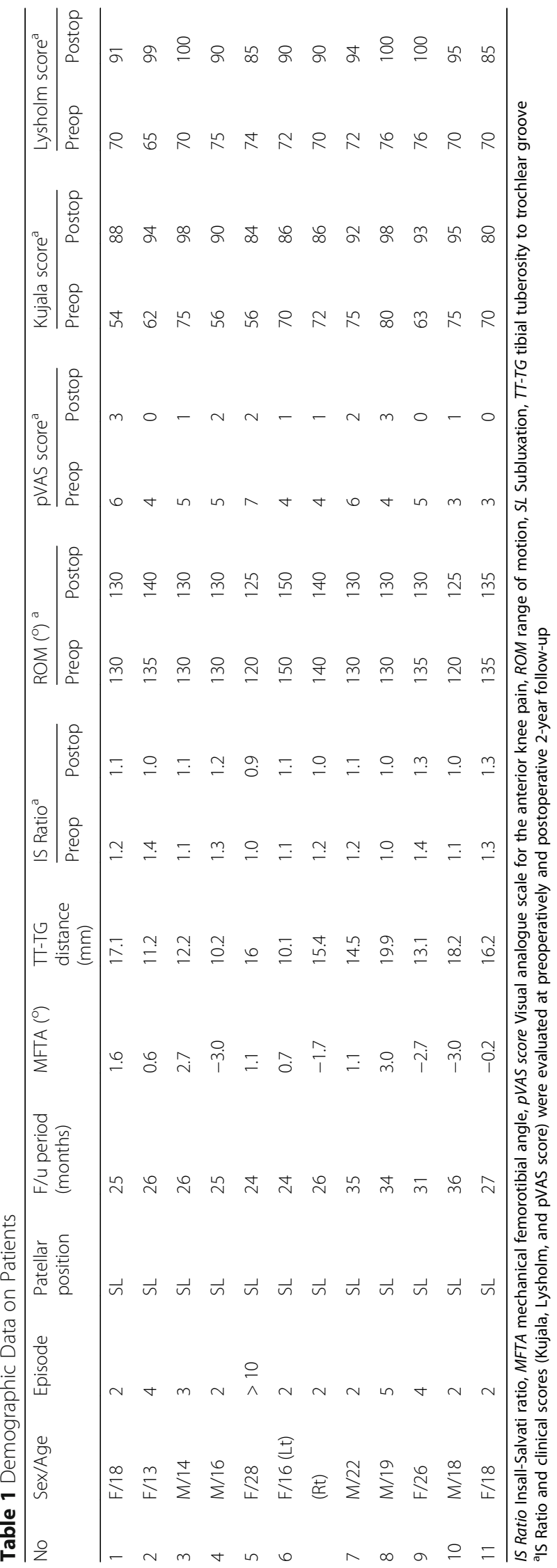


using the adjustable-length loop device (TightRope RT; Arthrex Inc., Naples, FL, USA). The diameter of all doubled tendons was 7 or $8 \mathrm{~mm}$ (Fig. 1).

\section{Preparation for patellar fixation}

A 2-cm incision was made at the medial border of the patella. The superomedial aspect of the patella was approached. To achieve aperture fixation on the patellar side, the free graft ends were directly fixed to the patella. A longitudinal periosteal incision was made about $1 \mathrm{~cm}$ lateral from the medial borderline of the patella, and the periosteum was detached and reflected medially. After the MPFL footprint was exposed, minimal decortication of the reconstruction area was performed for better boneto-graft healing. Two guidewires were drilled tangentially into the patella at the proximal and distal ends of the medial edge, and 2 suture anchors (Bio Mini-Revo; Linvatec, Largo, FL, USA) were inserted into the proximal margin and center of the medial aspect of the patella (Fig. 2). The free graft ends were sutured to the inserted grafts after flipping them over through the detached periosteum. Thereafter, the medial patellar periosteal tissue was sutured, covering the embedded graft and avoiding subcutaneous irritation by the knots.

\section{Femoral tunneling and graft tensioning}

In each femur, the femoral tunnel was made at the Schöttle point [29] in the proximal and anterior direction under $\mathrm{C}$-arm guidance, in order to prevent iatrogenic peroneal nerve injury (Fig. 3a). Then, the tunnel was created to pass the button of the adjustable-length loop device, for which a $4.0-\mathrm{mm}$ cannulated reamer was used. The length of the tunnel was measured using a depth gauge, and the femoral tunnel was drilled to have the same diameter as the graft until there was an 8-mm bone stock from the lateral femoral cortex (Fig. 3b). The graft was pulled into the femoral tunnel by using the lead suture inserted from the outside to the inside of the tunnel while maintaining equal tension on both bundles. The 2 strands of the graft passed between the first and second layers. The graft was fixed using the button of the device after it was flipped over the lateral cortex. After confirming that the button was on the cortex, manual traction of the suture loops was applied to fix the graft appropriately in full ROM of the knee joint with the lateral patellar edge positioned in line with the lateral trochlear border, under arthroscopic guidance (Fig. 4). The lateral retinaculum was released if the patient experienced lateral tightness. By using electrocautery under arthroscopic visualization, the capsular structure was released longitudinally along the lateral margin of the patella. The release was performed from $2 \mathrm{~cm}$ proximal to the superior patellar pole and extended distally for 1.5 to $2 \mathrm{~cm}$.

\section{Postoperative rehabilitation}

Tolerable weight bearing was allowed and quadriceps setting exercises could be started immediately with free $\mathrm{ROM}$, if tolerated. Running or cycling was permitted at 6 weeks after the operation; full activity was permitted at 3 months after the operation.

\section{Outcome evaluation}

Clinical outcomes related to recurrence of dislocation, apprehension test, ROM, clinical scores (Kujala score [30], Lysholm score [31], and visual analogue scale for anterior knee pain [pVAS]), complications, and radiological outcomes (congruence angle, lateral patellofemoral angle [32], and IS ratio) were assessed before and after the operation, and at the final 2-year follow-up.

Our primary outcomes of interest were radiographic parameters indicating patellar position, including congruence angle [2] and lateral patellofemoral angle [32]. The sulcus angle is the angle between a line between the lateral femoral condyle and intercondylar sulcus midpoint and a line between the medial femoral condyle and intercondylar sulcus midpoint. Each parameter was measured at 4 different angles of knee flexion $\left(30^{\circ}, 45^{\circ}, 60^{\circ}\right.$, and $\left.90^{\circ}\right)$.

\section{Statistical considerations}

Statistical tests were performed using IBM SPSS version 22 (IBM, Armonk, NY, USA). Continuous variables were described as mean \pm standard deviation (SD). A priori power analysis was performed to determine the sample size with the 2-sided hypothesis test considering an $\alpha$ error of 0.05 and power of 0.90 . The calculations involving our sample size of 11 patients indicated adequate power $(0.85-0.95)$ to detect a significant difference of 5 degrees in

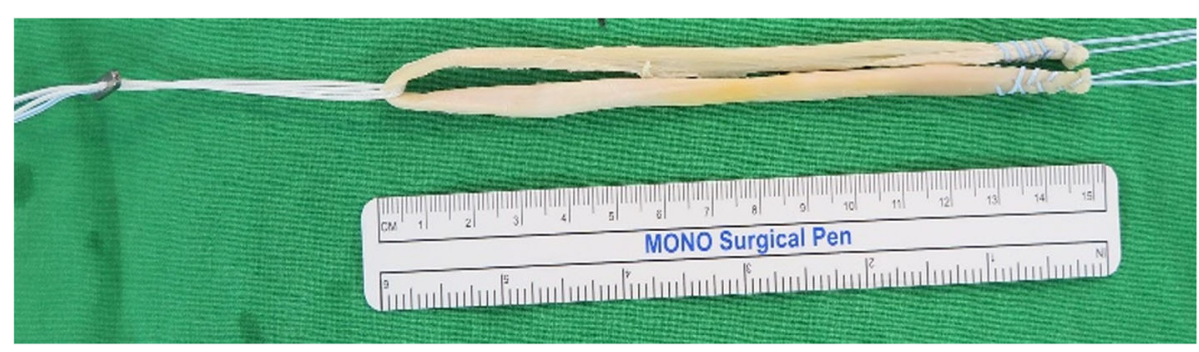

Fig. 1 Graft preparation 


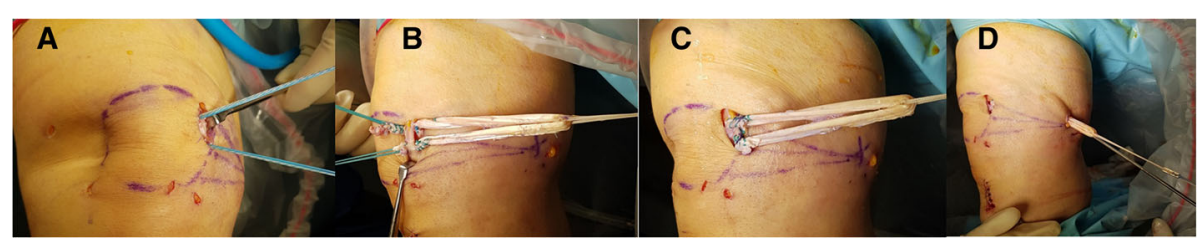

Fig. 2 A 2-cm longitudinal incision was made at the medial border of the patella. The deep fascia and periosteum were detached and reflected medially. After minimal decortication of the reconstruction area, 2 suture anchors were inserted into the proximal margin and center of the medial aspect of the patella (a). The free graft ends were sutured to the inserted grafts after flipping them over through the detached periosteum (b and $\mathbf{c}$ ). Looped graft with the adjustable-length loop device at the ending was brought into the separated layer between the vastus medialis obliquus (VMO) and the joint capsule, using long curved Kelly with caution to avoid any injury to the joint (d)

the measurement outcomes of congruence angle and lateral patellofemoral angle in the present study. Wilcoxon signed-rank tests were used to compare the pVAS, Lysholm, and Kujala scores; IS ratio; congruence angle; and lateral patellofemoral and sulcus angles before and after the operation. A $P$-value of $<0.05$ was considered statistically significant. The intra-class correlation coefficient (ICC) was determined to rule out observation bias between the 2 separate orthopedic surgeons. The parameters were measured twice, at an interval of 2 weeks.

\section{Results}

The patients' mean age at surgery was $18.6 \pm 4.4$ years (range: 13-28 years). The median follow-up period was 28.8 months (range: $24-48$ months). The average value of the IS ratio was 1.2 (SD: 0.1) and the TT-TG distance was $14.5 \mathrm{~cm}$ (SD: 3.2). All measured ICCs were almost good to perfect, ranging from 0.726 to 0.991 .

At the time of the final assessment, ROM was restored to the preoperative level and anterior knee pain improved in all patients, indicated by a decrease in the mean pVAS score from $4.7 \pm 1.2$ to $1.3 \pm 1.1(P<0.001)$. No patients experienced surgical complications, including patellar fracture and re-dislocation. The patellar instability symptoms improved, as confirmed on the basis of the radiographic outcomes as well as the Lysholm and Kujala scores. The mean Lysholm score improved from $71.7 \pm 3.2$ preoperatively to $93.3 \pm 5.6$ postoperatively $(P<0.001)$, and the Kujala score improved from $67.3 \pm 8.8$ preoperatively to $90.3 \pm 5.7$ postoperatively $(P<0.001)$ (Table 1$)$.

On merchant view with the patient supine, the knees flexed 30 degrees [33], the preoperative lateral patellofemoral angle $(-7.6 \pm 10.6$ to $7.6 \pm 3.1, p<0.001)$ and congruence angle $(30.1 \pm 13.9$ to $3.6 \pm 1.5, p<0.001)$ were improved after reconstruction. The congruence angle and the lateral patellofemoral angle were also improved significantly at 4 different flexion angles $\left(30^{\circ}\right.$, $45^{\circ}, 60^{\circ}$, and $\left.90^{\circ}\right)$ of the knee joint $(p<0.001)$. The improvements in these angles were maintained with no significant differences at the 4 different flexion angles (Table 2) (Fig. 5). Analysis of the measured radiographic parameters showed that patellar height, determined from the IS ratio, decreased slightly from $1.2 \pm 0.1$ to $1.1 \pm 0.1$ after the operation $(P<0.001)$.

\section{Discussion}

The most important findings of the present study were that the aperture fixation technique using the adjustable-length loop device from femoral cortical suspension systems improved the alignment parameters (congruence angle and

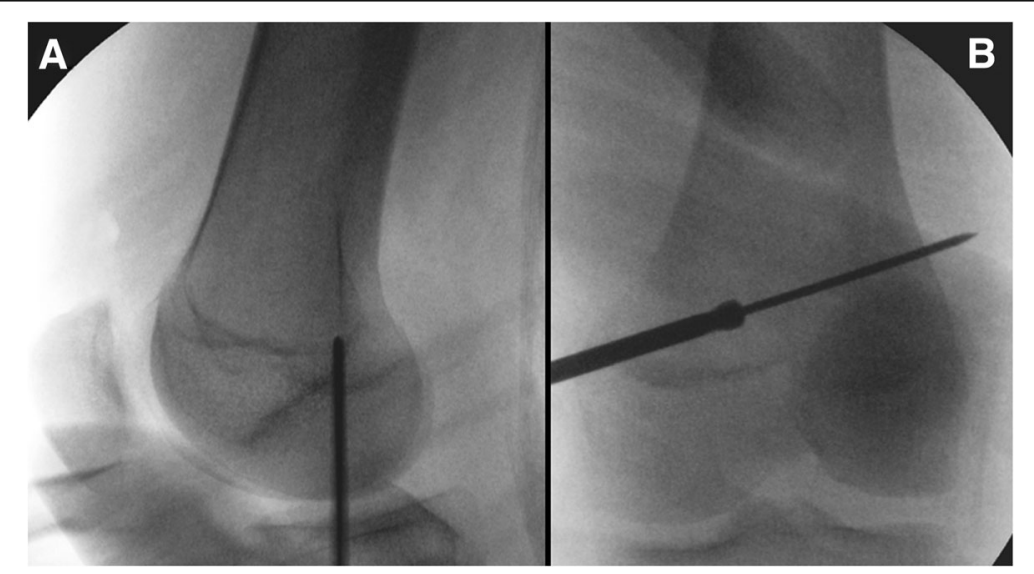

Fig. 3 A femoral tunnel was made at the Schöttle point [2] (a) in the proximal and anterior direction in order to prevent iatrogenic peroneal nerve injury. $\mathbf{b}$ Intraoperative control was achieved using an image intensifier 

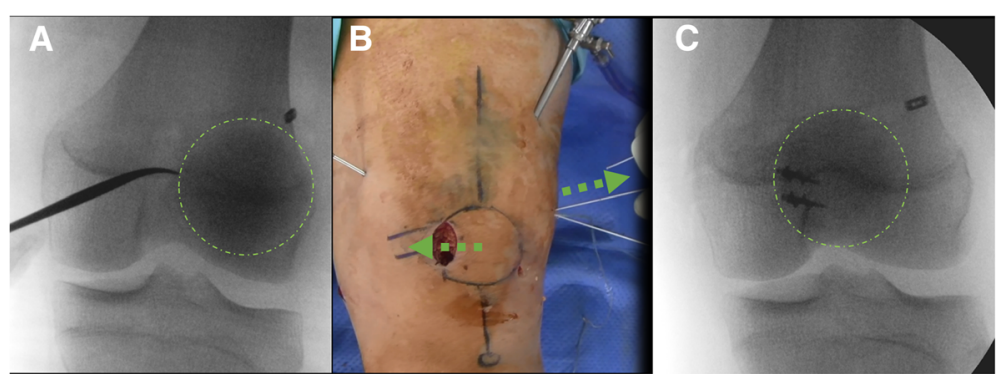

Fig. 4 The patellar position was tracked and fixed simultaneously by manual traction of the suture loops (b), and the graft was appropriately fixed in full range of motion of the knee joint with the lateral patellar edge positioned in line with the lateral trochlear border, under arthroscopic guidance $(\mathbf{a}, \mathbf{c})$

Table 2 Preoperative, Immediate Postoperative, and Two-year Follow-Up Radiologic Parameters ${ }^{a}$

\begin{tabular}{|c|c|c|c|c|c|c|}
\hline \multicolumn{2}{|l|}{ Knee flexion angle } & \multicolumn{2}{|l|}{ Preop } & \multicolumn{2}{|l|}{ Postop } & \multirow{2}{*}{$\frac{F / U}{8.8 \pm 4.1}$} \\
\hline Lateral patellofemoral angle $\left(^{\circ}\right)$ & $30^{\circ}$ & $-7.6 \pm 10.6$ & & $7.6 \pm 3.1$ & & \\
\hline & & & $<0.001$ & & 0.260 & \\
\hline & $45^{\circ}$ & $-6.2 \pm 6.5$ & & $9.2 \pm 0.4$ & & $10.4 \pm 4.6$ \\
\hline & & & 0.001 & & 0.285 & \\
\hline & $60^{\circ}$ & $-0.9 \pm 5.6$ & & $11.0 \pm 2.5$ & & $13.0 \pm 3.2$ \\
\hline & & & 0.006 & & 0.140 & \\
\hline & $90^{\circ}$ & $6.6 \pm 3.4$ & & $12.2 \pm 2.4$ & & $15.6 \pm 3.2$ \\
\hline & & & 0.033 & & 0.093 & \\
\hline \multirow[t]{8}{*}{ Congruence angle $\left({ }^{\circ}\right)$} & $30^{\circ}$ & $30.1 \pm 13.9$ & & $3.6 \pm 1.5$ & & $2.9 \pm 1.3$ \\
\hline & & & $<0.001$ & & 0.221 & \\
\hline & $45^{\circ}$ & $26.1 \pm 15.2$ & & $2.9 \pm 1.0$ & & $2.7 \pm 1.0$ \\
\hline & & & 0.001 & & 0.233 & \\
\hline & $60^{\circ}$ & $18.4 \pm 11.0$ & & $2.8 \pm 1.6$ & & $2.7 \pm 1.4$ \\
\hline & & & 0.014 & & 0.008 & \\
\hline & $90^{\circ}$ & $11.5 \pm 7.4$ & & $1.7 \pm 1.1$ & & $1.7 \pm 1.0$ \\
\hline & & & 0.010 & & 0.483 & \\
\hline \multirow[t]{8}{*}{ Sulcus angle $\left({ }^{\circ}\right)$} & $30^{\circ}$ & $146.9 \pm 7.4$ & & $145.3 \pm 6.4$ & & $145.4 \pm 5.5$ \\
\hline & & & 0.491 & & 0.442 & \\
\hline & $45^{\circ}$ & $146.6 \pm 2.4$ & & $145.5 \pm 1.7$ & & $146.0 \pm 4.8$ \\
\hline & & & 0.549 & & 0.255 & \\
\hline & $60^{\circ}$ & $146.1 \pm 4.5$ & & $145.2 \pm 5.9$ & & $146.5 \pm 5.4$ \\
\hline & & & 0.870 & & 0.302 & \\
\hline & $90^{\circ}$ & $146.4 \pm 3.4$ & & $145.8 \pm 5.5$ & & $144.8 \pm 5.0$ \\
\hline & & & 0.634 & & 0.322 & \\
\hline \multirow[t]{2}{*}{ IS Ratio } & & $1.2 \pm 0.1$ & & & & $1.1 \pm 0.1$ \\
\hline & & & & $<0.001$ & & \\
\hline
\end{tabular}




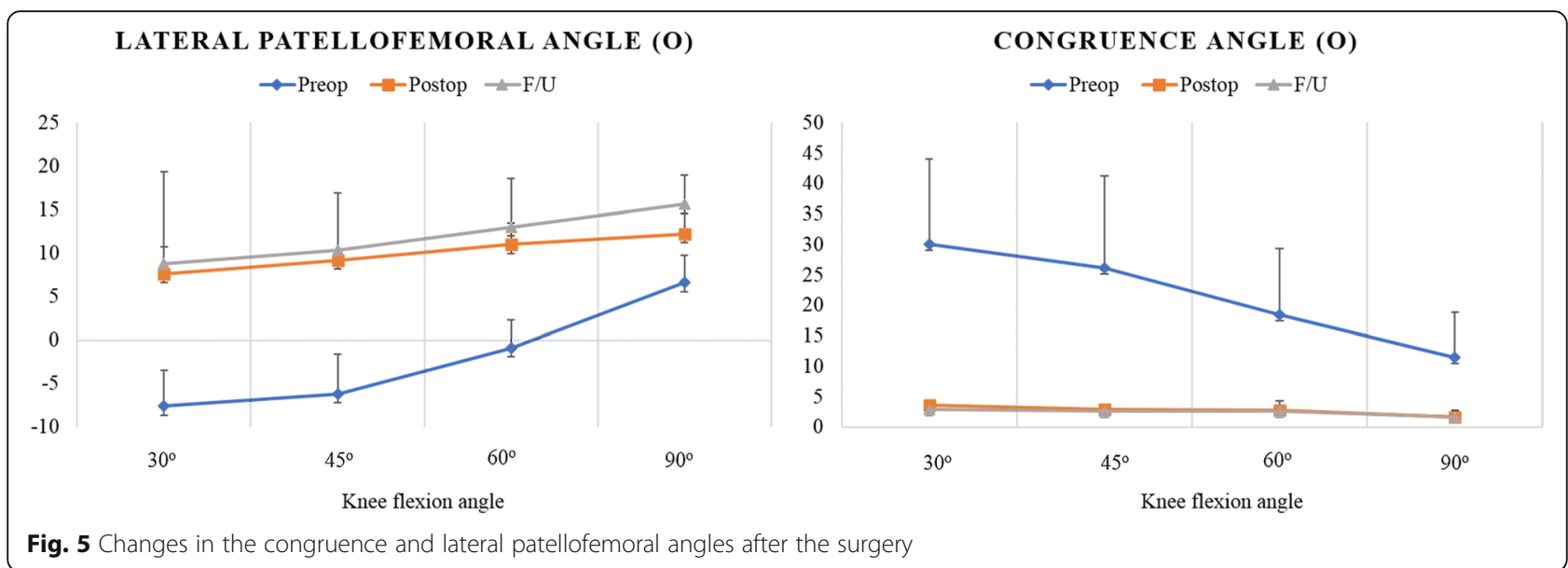

patellar tilt angle) and yielded high stability in full ROM of the knee joint for 2 years after the operation.

Reconstruction techniques with the DB structure for restoring the anatomic shape of the MPFL have been recently highlighted $[34,35]$. The MPFL is not a singlebundle structure but a complex of functionally varying fibers, some of which are taut and some are slack, throughout the range of knee motion [19]. Superior patellar fixation may cause patellar instability at mid to high knee angles, and conversely, inferior patellar fixation may produce excessive laxity at low flexion angles [19]. Anatomic DB-MPFLR lowers patellar rotation during the flexionextension movement that may occur during single-bundle reconstruction.

Undoubtedly, the aperture fixation technique has clinical benefits in terms of the effort required to decrease the risk of delayed or insufficient tendon-to-bone healing [21, 24]. Schöttle et al. indicated that it provides high stability, as the proximal bundle seems to stabilize in extension while the distal bundle stabilizes in flexion.

However, as described in the Introduction, we hypothesized that certain reciprocal movement of the looped graft into the femoral tunnel may ensure more biocompatible reconstruction, rather than leaving the tension of each bundle to the discretion of the surgeon without a thorough understanding of the length change or the biomechanics of the 2 bundles of the MPFL throughout the range of knee motion. We did not directly evaluate the permissible amount of the reciprocal movement of the looped graft into the femoral tunnel. We sought to determine the clinical significance of the technique on the basis of our finding that improvements in radiographic parameters representing patellar position were noted irrespective of the angle of knee flexion $\left(30^{\circ}, 45^{\circ}, 60^{\circ}\right.$, or $\left.90^{\circ}\right)$. We also found that at the 2-year follow-up, no graft slackening, graft failure, or reduction failure from elongation of the graft or the "bungee" effect in the femoral socket had occurred in any case. However, it remains to be determined whether a kinematic isometric length change of about $6-$ $9 \mathrm{~mm}$ throughout the range of knee motion [19] affects the radiological and clinical outcomes in clinical practice.

Other studies described several techniques for anatomic DB-MPFLR. White and Sherman [17] used an absorbable soft tissue interference screw for femoral fixation in $30^{\circ}$ of knee flexion. Colvin and West [36] also used absorbable interference screws for femoral fixation of the 2 free ends of the graft in $30^{\circ}$ of knee flexion, and performed combined bone groove and suture anchor fixation at the patella. Dejour et al. [37] used a "Y"-shaped graft for reconstruction. An absorbable soft tissue interference screw was used for femoral fixation, and the lateral bone bridge tie was used for patellar fixation. Additionally, they introduced the surgical technique of lateral retinaculum plasty to release eccentric dynamic loading through the ROM.

Graft fixation is recommended with appropriate tension at $20-30^{\circ}$ of knee flexion and with the patella aligned in the trochlear groove throughout the entire ROM of the knee. However, it is not easy to determine the appropriate graft tension during graft fixation. Previous arbitrary techniques that rely on the surgeon's subjective skill carry the risk of over-tensioning of the MPFL graft, which can increase the patellofemoral pressure [38]. Goutallier et al. [39] reported that anterior knee pain persisted after the operation in up to $38-40 \%$ of patients. Our technique using the adjustablelength loop device has clinical applicability in this aspect. The patellar position, which can easily be determined under arthroscopic guidance throughout flexion, was tracked and fixed simultaneously by a simple pulling of the suture loops. Moreover, the possible reciprocal movement of the looped graft into the femoral tunnel might release eccentric loading through the ROM. It can also diminish the learning curve to achieve appropriate graft tensioning during MPFL reconstruction. In the present study, we found that anterior knee pain was relieved in all patients, the patients had full ROM immediately after the surgery, and there was no reduction loss at follow-up with early rehabilitation. 
Our study has some inherent limitations because of its retrospective design. The relatively short follow-up period and small sample size were also limitations in judging the postoperative outcomes. However, a 2-year follow-up period is sufficient to determine clinical outcomes such as re-dislocation. Furthermore, a lack of comparison with other aperture techniques is a major limitation of the present study. In addition, this study did not directly compare the patellofemoral kinematic changes by using additional biomechanical measurement tools. Thus, the findings may not be adequate to determine the clinical relevance of using the adjustable-length loop device with regard to patellofemoral kinematics.

\section{Conclusion}

New DB technique with aperture fixation at the patella and femur by using an adjustable-length loop device offers high stability with full ROM of the knee joint, can be considered as a feasible procedure and technique for recurrent patellar dislocation.

\section{Abbreviations}

ACLR: Anterior cruciate ligament reconstruction; AP: Anteroposterior; DBMPFLR: Double-bundle medial patellofemoral ligament reconstruction; ICC: Intra-class correlation coefficient; MFTA: Mechanical femorotibial angle; MPFL: Medial patellofemoral ligament; PVAS: Pain visual analogue scale; ROM: Range of motion; RPD: Recurrent patellar dislocation; SD: Standard deviation; TT-TG: Tibial tuberosity to trochlear groove

\section{Acknowledgments}

The authors thank all members of the Sports Medicine Center, Gil Hospital, for their great scientific debates.

\section{Availability of data and materials}

The datasets used and/or analyzed during the current study are available from the corresponding author on reasonable request.

\section{Authors' contributions}

JAS and BHL participated in the study design and helped in drafting the manuscript. BHL, as a statistical consultant, performed the statistical analysis. JAS and JKL conceived the study, participated in its design and coordination, and helped draft the manuscript. All authors participated in the development of and approved the final manuscript, and agreed to be accountable for the integrity of the content.

\section{Ethics approval and consent to participate}

All procedures performed in studies involving human participants were in accordance with the ethical standards of the institutional and/or national research committee and with the 1964 Helsinki Declaration and its later amendments or comparable ethical standards. The current study obtained Institutional Review Board approval from our institution (Gachon University of Medicine and Science, GA IRB 2017-236) before study onset, and our protocol was also approved. Informed consent was obtained from all patients. All patients provided written informed consent for participation. For adolescent participants, written informed consent was obtained from their parents/guardians.

\section{Consent for publication}

Not applicable.

\section{Competing interests}

The authors declare that they have no competing interests.

\section{Publisher's Note}

Springer Nature remains neutral with regard to jurisdictional claims in published maps and institutional affiliations.

\section{Author details}

'Department of Orthopaedic Surgery, Gil Hospital, Gachon University of Medicine and Science, Inchon, South Korea. ${ }^{2}$ Department of Orthopedic Surgery, Kang-Dong Sacred Heart Hospital, Hallym University Medical School, 134-701, Gil-dong, Seoul, South Korea.

Received: 25 September 2017 Accepted: 16 September 2018 Published online: 25 September 2018

\section{References}

1. White BJ, Sherman OH. Patellofemoral instability. Bull NYU Hosp Jt Dis. 2009; 67:22-9.

2. Colvin AC, West RV. Patellar instability. J Bone Joint Surg Am. 2008;90:2751-62.

3. Dejour $H$, Walch $G$, Neyret P, Adeleine P: [dysplasia of the femoral trochlea]. Rev Chir Orthop Reparatrice Appar Mot 1990, 76:45-54.

4. Dejour H, Walch G, Nove-Josserand L, Guier C. Factors of patellar instability: an anatomic radiographic study. Knee Surg Sports Traumatol Arthrosc. 1994;2:19-26.

5. Goutallier D, Bernageau J, Lecudonnec B. the measurement of the tibial tuberosity. Patella groove distanced technique and results (author's transl). Rev Chir Orthop Reparatrice Appar Mot. 1978;64:423-8.

6. Geenen E, Molenaers G, Martens M. Patella alta in patellofemoral instability. Acta Orthop Belg. 1989;55:387-93.

7. Neyret P, Robinson AH, Le Coultre B, Lapra C, Chambat P. Patellar tendon length--the factor in patellar instability? Knee. 2002;9:3-6.

8. Simmons E Jr, Cameron JC. Patella Alta and recurrent dislocation of the patella. Clin Orthop Relat Res. 1992;(274):265-9.

9. Ward SR, Terk MR, Powers CM. Patella Alta: association with patellofemoral alignment and changes in contact area during weight-bearing. J Bone Joint Surg Am. 2007;89:1749-55.

10. Guerrero P, Li X, Patel K, Brown M, Busconi B. Medial patellofemoral ligament injury patterns and associated pathology in lateral patella dislocation: an MRI study. Sports Med Arthrosc Rehabil Ther Technol. 2009;1:17.

11. Conlan T, Garth WP,J, Lemons JE. Evaluation of the medial soft-tissue restraints of the extensor mechanism of the knee. J Bone Joint Surg Am. 1993;75:682-93.

12. Burks RT, Desio SM, Bachus KN, Tyson L, Springer K. Biomechanical evaluation of lateral patellar dislocations. Am J Knee Surg. 1998;11:24-31.

13. Buckens CF, Saris DB. Reconstruction of the medial patellofemoral ligament for treatment of patellofemoral instability: a systematic review. Am J Sports Med. 2010;38:181-8.

14. Christiansen SE, Jacobsen BW, Lund B, Lind M. Reconstruction of the medial patellofemoral ligament with gracilis tendon autograft in transverse patellar drill holes. Arthroscopy. 2008;24:82-7.

15. Han H, Xia Y, Yun X, Wu M. Anatomical transverse patella double tunnel reconstruction of medial patellofemoral ligament with a hamstring tendon autograft for recurrent patellar dislocation. Arch Orthop Trauma Surg. 2011;131:343-51.

16. Panni AS, Alam M, Cerciello S, Vasso M, Maffulli N. Medial patellofemoral ligament reconstruction with a divergent patellar transverse 2-tunnel technique. Am J Sports Med. 2011;39:2647-55.

17. Schottle PB, Fucentese SF, Romero J. Clinical and radiological outcome of medial patellofemoral ligament reconstruction with a semitendinosus autograft for patella instability. Knee Surg Sports Traumatol Arthrosc. 2005;13:516-21.

18. Amis AA, Firer P, Mountney J, Senavongse W, Thomas NP. Anatomy and biomechanics of the medial patellofemoral ligament. Knee. 2003;10:215-20.

19. Song SY, Pang CH, Kim CH, Kim J, Choi ML, Seo YJ. Length change behavior of virtual medial patellofemoral ligament fibers during in vivo knee flexion. Am J Sports Med. 2015:43:1165-71.

20. Wang JH, Kato Y, Ingham SJ, Maeyama A, Linde-Rosen M, Smolinski P, Fu $\mathrm{FH}$. Measurement of the end-to-end distances between the femoral and tibial insertion sites of the anterior cruciate ligament during knee flexion and with rotational torque. Arthroscopy. 2012;28:1524-32.

21. Schottle PB, Schmeling A, Rosenstiel N, Weiler A. Radiographic landmarks for femoral tunnel placement in medial patellofemoral ligament reconstruction. Am J Sports Med. 2007;35:801-4. 
22. Brand J Jr, Weiler A, Caborn DN, Brown CH Jr, Johnson DL. Graft fixation in cruciate ligament reconstruction. Am J Sports Med. 2000;28:761-74.

23. Steensen RN, Dopirak RM, WG MD 3rd. The anatomy and isometry of the medial patellofemoral ligament: implications for reconstruction. Am J Sports Med. 2004;32:1509-13.

24. Li J, Li Y, Wei J, Wang J, Gao S, Shen Y. A simple technique for reconstruction of medial patellofemoral ligament with bone-fascia tunnel fixation at the medial margin of the patella: a 6-year-minimum follow-up study. J Orthop Surg Res. 2014;9:66

25. Lubowitz JH, Schwartzberg R, Smith P. Cortical suspensory button versus aperture interference screw fixation for knee anterior cruciate ligament softtissue allograft: a prospective, randomized controlled trial. Arthroscopy. 2015;31:1733-9.

26. Dejour D, Le Coultre B. Osteotomies in patello-femoral instabilities. Sports Med Arthrosc. 2007:15:39-46.

27. Shabshin N, Schweitzer ME, Morrison WB, Parker L. MRI criteria for patella Alta and Baja. Skelet Radiol. 2004;33:445-50.

28. Haviv B, Bronak $S$, Thein $R$, Thein $R$. The results of corrective osteotomy for valgus arthritic knees. Knee Surg Sports Traumatol Arthrosc. 2013;21:49-56.

29. Barnett AJ, Howells NR, Burston BJ, Ansari A, Clark D, Eldridge JD. Radiographic landmarks for tunnel placement in reconstruction of the medial patellofemoral ligament. Knee Surg Sports Traumatol Arthrosc. 2012;20:2380-4

30. Kujala UM, Jaakkola LH, Koskinen SK, Taimela S, Hurme M, Nelimarkka O. Scoring of patellofemoral disorders. Arthroscopy. 1993;9:159-63.

31. Tegner Y, Lysholm J. Rating systems in the evaluation of knee ligament injuries. Clin Orthop Relat Res. 1985;(198):43-9.

32. Lee CH, Wu CC, Pan RY, Lu HT, Shen HC. Medial retinacular flap advancement and arthroscopic lateral release for symptomatic chronic patellar lateral subluxation with tilting. Knee Surg Sports Traumatol Arthrosc. 2014;22:2499-504.

33. Laurin CA, Levesque HP, Dussault R, Labelle H, Peides JP. The abnormal lateral patellofemoral angle: a diagnostic roentgenographic sign of recurrent patellar subluxation. J Bone Joint Surg Am. 1978;60:55-60.

34. Schottle PB, Romero J, Schmeling A, Weiler A. Technical note: anatomical reconstruction of the medial patellofemoral ligament using a free gracilis autograft. Arch Orthop Trauma Surg. 2008;128:479-84.

35. Thaunat M, Erasmus PJ. The favourable anisometry: an original concept for medial patellofemoral ligament reconstruction. Knee. 2007;14:424-8,

36. Wang HD, Dong JT, Gao SJ. Medial patellofemoral ligament reconstruction using a bone groove and a suture anchor at patellar: a safe and firm fixation technique and 3-year follow-up study. J Orthop Surg Res. 2016;11:138.

37. Niu Y, Wang X, Liu C, Wang X, Dong Z, Niu J, Wang F. Double-bundle anatomical medial patellofemoral ligament reconstruction with lateral retinaculum plasty can lead to good outcomes in patients with patellar dislocation. Knee Surg Sports Traumatol Arthrosc. 2017.

38. Stephen JM, Lumpaopong P, Dodds AL, Williams A, Amis AA. The effect of tibial tuberosity medialization and lateralization on patellofemoral joint kinematics, contact mechanics, and stability. Am J Sports Med. 2015;43:186-94.

39. Feller JA, Richmond AK, Wasiak J. Medial patellofemoral ligament reconstruction as an isolated or combined procedure for recurrent patellar instability. Knee Surg Sports Traumatol Arthrosc. 2014;22:2470-6.

Ready to submit your research? Choose BMC and benefit from:

- fast, convenient online submission

- thorough peer review by experienced researchers in your field

- rapid publication on acceptance

- support for research data, including large and complex data types

- gold Open Access which fosters wider collaboration and increased citations

- maximum visibility for your research: over $100 \mathrm{M}$ website views per year

At $\mathrm{BMC}$, research is always in progress.

Learn more biomedcentral.com/submissions 